\title{
Nouveautés liées à Via sicura
}

Direction et gestion du Centre Suisse de formation pour les expertises en aptitude à la conduite automobile

Avec le volet de Via sicura qui prend effet au 1.7.2016, des modifications qui affectent directement les médecins sont prévues. Aux fins d'assurance qualité, les médecins qui réalisent les examens d'aptitude à la conduite devront à l'avenir remplir certaines conditions et suivre des formations. La Société Suisse de Médecine Légale (SSML) a été chargée de dispenser ces dernières.

\section{Introduction}

Depuis l'adoption du programme d'action visant à renforcer la sécurité routière Via sicura le 15.6.2012, différentes mesures sont progressivement entrées en vigueur. Du point de vue des médecins, les modifications de l'art. 15d de la loi sur la circulation routière (LCR) sont les plus importantes à ce jour.

Les autorités ne sont autorisées à confier des mandats de détermination de l'aptitude à la conduite qu'aux médecins possédant les qualifications requises.

Outre la définition des infractions qui entraînent une détermination de l'aptitude à la conduite en cas de délit lié à l'alcool ou à la drogue, il a été clairement stipulé que les médecins sont libérés du secret professionnel lors des communications aux autorités. Il a également été stipulé qu'en cas de communication d'un office AI sur l'existence de doutes concernant l'aptitude à la conduite d'une personne, une enquête devra avoir lieu [1].

En outre, depuis le 1.7.2014, la consigne pour les délits qui soulèvent des doutes quant à l'aptitude à la conduite d'une personne est que ces enquêtes ne peuvent être réalisées que par des médecins et psychologues adéquatement qualifiés, à savoir un spécialiste en médecine du trafic SSML en cas de questions relevant de la médecine du trafic et un psychologue spécialiste en psychologie de la circulation FSP en cas de questions relevant de la psychologie du trafic [2].

Des exigences médicales minimales adaptées entreront en vigueur le 1.7.2016. En même temps, les conditions que doivent remplir les médecins qui souhaitent à l'avenir déterminer l'aptitude à la conduite sont définies aux fins d'assurance qualité. Ainsi, les médecins comme leurs patients sont directement concernés. Les autorités ne sont autorisées à confier des mandats de détermination de l'aptitude à la conduite qu'aux médecins possédant les qualifications requises, respectivement à n'accepter que les évaluations de ces derniers.

\section{Exigences médicales minimales: Annexe 1 à l'ordonnance réglant l'admis- sion à la circulation routière (OAC)}

A l'avenir, il n'y aura plus que deux groupes médicaux dans l'UE:

- Groupe 1: catégories de permis de conduire A et B, sous-catégories A1 et B1, catégories spéciales F, G et $M$

- Groupe 2: catégories de permis de conduire C et D, sous-catégories $\mathrm{C} 1$ et D1, autorisation de transporter des personnes à titre professionnel, experts de la circulation, moniteurs de conduite

Les exigences minimales actuelles, qui datent des années 1970, ont été revues et adaptées à une nomenclature moderne. Certaines exigences existantes ont été supprimées, d'autres reformulées. Certaines exigences minimales n'ont été adaptées que de manière ponctuelle. En ce qui concerne les facultés visuelles, il y a eu un rapprochement avec les directives de l'UE et pour le diabète sucré, des règles détaillées ont été fixées. Certaines directives ont déjà été établies avec les sociétés professionnelles compte tenu des nouvelles exigences minimales.

\section{Assurance qualité de l'évaluation de l'aptitude à la conduite}

Les conditions à remplir par les médecins pour déterminer l'aptitude à la conduite ont été inscrites dans la loi et quatre niveaux de qualification au total ont été définis pour la médecine du trafic [3, 4]. En principe, les 


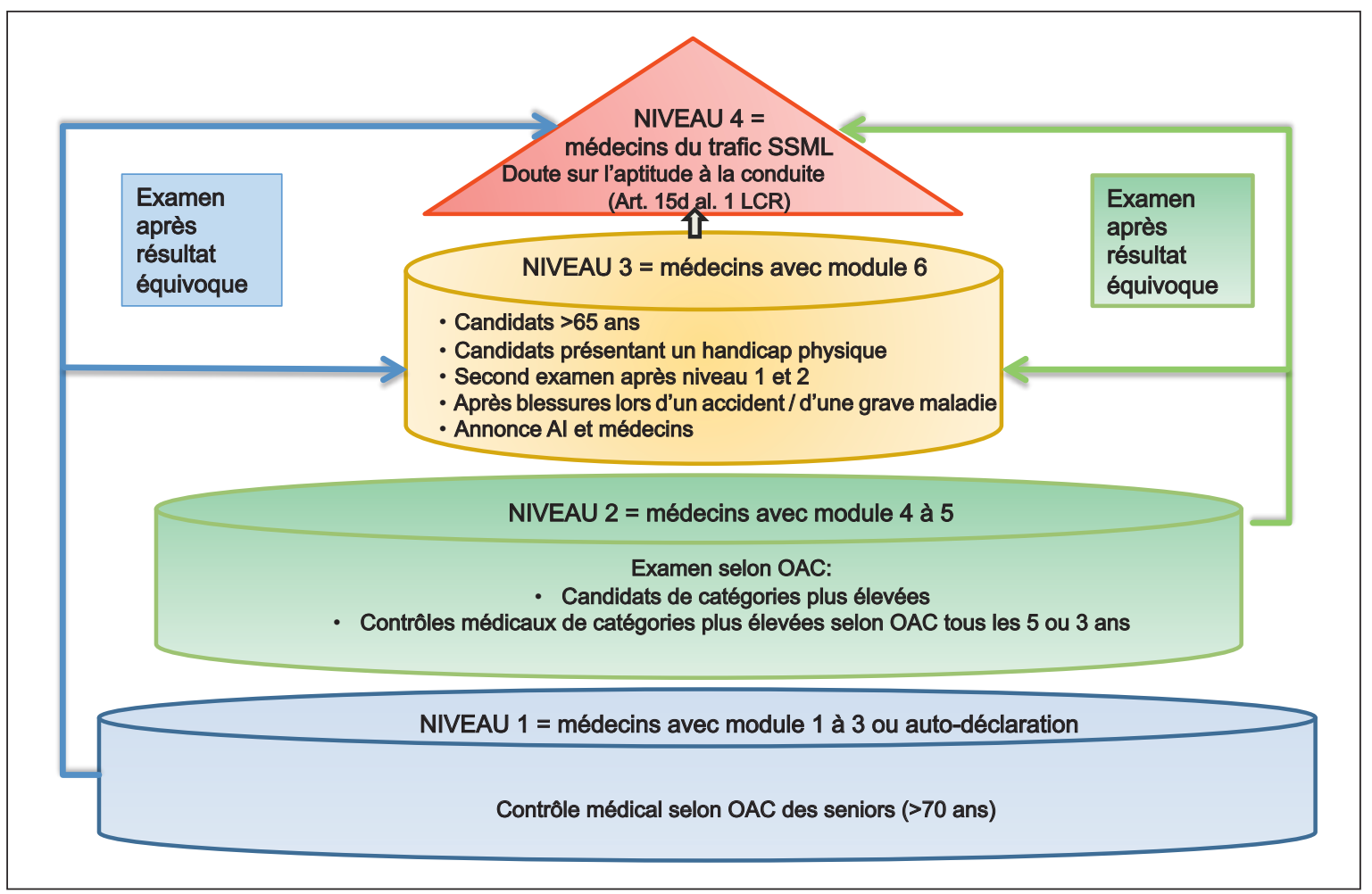

Figure 1: Le modèle progressif.

différents médecins doivent posséder un titre postgrade fédéral ou un titre postgrade étranger reconnu.

Chaque niveau autorise à pratiquer des examens spécifiques, sachant que le niveau supérieur comprend également l'autorisation de réaliser les examens de niveau inférieur [4]. La définition des conditions à remplir par le médecin pour les différentes autorisations devrait uniformiser la qualité et l'évaluation dans toute la Suisse.

A l'avenir, tout médecin possédant le niveau de qualification adéquat pourra réaliser les examens correspondants, alors que jusqu'ici, ceci était dans certains cantons réservé à des médecins désignés par le service des automobiles (par ex. médecins officiels, de district ou conseils).

En cas de résultat ambigu, le médecin peut conseiller au service des automobiles de faire évaluer l'aptitude à la conduite par un spécialiste dont le niveau de qualification est supérieur (fig. 1).

\section{Centre Suisse de formation pour les expertises en aptitude à la conduite automobile}

L'organisation et la réalisation des formations ont, sur la base de l'art. 5h OAC, été confiées à la Société Suisse de Médecine Légale (SSML). Le Centre Suisse de formation pour les expertises en aptitude à la conduite automobile, intégré à la SSML du point de vue organisationnel, garantit une formation homogène dans toute la Suisse. Des cours de formation pour tous les niveaux de qualification sont proposés. Les cours ont une structure modulaire et transmettent les compétences techniques requises pour réaliser les différents examens conformément aux normes de qualité courantes.

Pour chaque manifestation, une demande d'octroi de crédits est déposée auprès de l'association professionnelle SSMI/SSMG, en sorte que les cours proposés puissent être intégrés au curriculum de formation continue du médecin concerné.

\section{Enregistrement sur www.medtraffic.ch}

La collaboration de la SSML et de l'Association des services des automobiles (asa) a donné naissance à une plateforme (www.medtraffic.ch), sur laquelle les médecins peuvent s'enregistrer, s'inscrire à des cours, gérer leur formation initiale et continue en lien avec la détermination de l'aptitude à la conduite et trouver les principaux renseignements concernant les examens d'aptitude à la conduite. Les questions peuvent être envoyées à info[at]medtraffic.ch.

Tous les médecins qui souhaitent procéder à des examens d'aptitude à la conduite doivent s'enregistrer sur ce site Internet avec leur GLN (Global Location Number) et leur date de naissance. L'administration des données personnelles y sera alors également possible. La date de naissance est importante parce que le législateur a prévu que les médecins examinateurs ne pourront pas 
être âgés de plus de 70 ans. L'enregistrement devrait être possible à partir de novembre 2015.

Les services des automobiles ont également accès aux informations qui leur sont nécessaires et peuvent vérifier le niveau de qualification à réception d'un certificat, d'un rapport ou d'une expertise.

Les conducteurs eux-mêmes ont également la possibilité de rechercher sur cette plateforme des médecins présentant le niveau de qualification requis et de prendre rendez-vous auprès d'eux ou du service d'examen pour l'examen nécessaire. Comme les niveaux de qualification sont valables dans toute la Suisse, le conducteur peut également consulter un médecin dûment qualifié hors de son canton de domicile.

Les médecins qui n'ont pas suivi les modules de formation continue de la SSML n'apparaîtront toutefois pas sur la plateforme. Il en ira de même pour les médecins effectuant les examens conformément aux dispositions transitoires (voir «Dispositions transitoires»). Les services des automobiles cantonaux tiennent toutefois des listes pour leur canton et connaissent la qualification des médecins.

\section{Niveaux de qualification en médecine du trafic $[3,4]$}

\section{Niveau 1}

Habilité à déterminer l'aptitude à la conduite de conducteurs qui doivent se soumettre à un examen tous les 2 ans à partir de leurs 70 ans.

Les médecins doivent posséder les connaissances et aptitudes définies à l'annexe 1bis OAC:

a. connaissance et compréhension des bases juridiques pertinentes pour ce qui est des contrôles relevant de la médecine du trafic (LCR, OCCR, OCR, OAC, dispositions d'exécution cantonales);

b. connaissance des procédures administratives entre l'autorité cantonale et le médecin chargé de l'examen;

c. connaissance des indices d'inaptitude à la conduite pouvant amener à une évaluation relevant de la médecine du trafic ou nécessitant des examens complémentaires et des courses de contrôles supervisées par un médecin ainsi que de la procédure à suivre;

d. connaissance de la procédure d'examen;

e. capacité d'évaluer l'aptitude à la conduite selon les exigences médicales minimales dans les divers groupes de diagnostic et identification d'une consommation de substances problématiques;

f. connaissance des déficiences et maladies pouvant avoir des effets sur la conduite des personnes âgées de plus de 70 ans et capacité d'évaluer l'aptitude à la conduite, en particulier en présence de déficits cognitifs; g. connaissance des diverses directives médicales des associations professionnelles (par ex. les directives concernant l'aptitude à conduire lors de diabète sucré de la Société Suisse d'Endocrinologie et de Diabétologie) et aptitude à les utiliser;

h. connaissance des conditions pouvant être arrêtées par l'autorité cantonale;

i. aptitude à transmettre les informations requises aux autorités cantonales dans les règles (annexe 3 $\mathrm{OAC})$.

Les médecins sont libres d'acquérir ces compétences comme ils le souhaitent. Ils peuvent attester les posséder par le biais d'une auto-déclaration.

En soi, l'examen d'aptitude à la conduite ne pose pas de problème au médecin généraliste. On sait toutefois d'expérience que des erreurs de jugement sont fréquentes dans l'évaluation de l'aptitude à la conduite dans son contexte légal, parce que les exigences légales sont inconnues, parce qu'elles donnent lieu à des interprétations différentes, parce qu'il n'a pas été formulé de conditions alors que cela aurait été judicieux ou enfin parce que lors de la formulation des conditions, celles recommandées ne peuvent pas être mises en œuvre par les autorités.

La SSML propose un cours d'une journée (modules 1 à 3). Son objet principal est la transmission des connaissances prescrites par la loi. Un document pouvant être utilisé pour l'évaluation de l'aptitude à la conduite est remis lors du cours.

Même s'il n'y a pas d'obligation légale de suivre un tel cours, cette formation d'une journée permet d'acquérir des connaissances étendues, susceptibles d'être utiles en cas de litige judiciaire avec la personne concernée ou les autorités. D’autant plus que les connaissances juridiques et le raisonnement forensique ne jouent pas un rôle central dans la pratique quotidienne, mais sont déterminants en lien avec le rôle d'expert du médecin lors de la détermination de l'aptitude à la conduite.

\section{Niveau 2}

Habilité à déterminer l'aptitude à la conduite de candidats à un permis d'élève conducteur ou à un permis de conduire de catégorie plus élevée.

Un cours d'une journée (modules 4 et 5) est obligatoire pour le niveau 2. La SSML recommande toutefois vivement aux médecins qui souhaitent obtenir un niveau de qualification supérieur de suivre au préalable les modules 1 à 3 , car ces niveaux plus élevés comportent également des exigences plus strictes vis-à-vis du médecin examinateur et requièrent une solide formation de base, notamment en termes de réglementations légales. 


\section{Niveau 3}

Habilité les médecins à déterminer l'aptitude à la conduite de personnes dont le premier examen de niveau 1 ou 2 a donné un résultat ambigu, à effectuer des examens dans les cas visés à l'art. 15d, al.1, let. d et e (par ex. personnes handicapées physiquement, communication par le service AI). Les niveaux 1 et 2 sont indispensables.

La formation au niveau 3, qui permet la réalisation de certaines expertises, nécessite un cours supplémentaire d'une $1 / 2$-journée (module 6), puis la réalisation de 5 expertises sous la supervision d'un spécialiste en médecine du trafic SSML.

\section{Niveau 4 = spécialiste en médecine du trafic SSML}

Habilite les médecins à réaliser l'ensemble des examens d'aptitude à la conduite. La condition requise est la formation de spécialiste en médecine du trafic SSML [5].

\section{La formation continue d'une journée permet d'acquérir des connaissances étendues.}

\section{Reconnaissance de la qualification en médecine du trafic}

La reconnaissance est délivrée par l'autorité du canton dans lequel le médecin travaille le plus souvent. Elle est valable dans toute la Suisse pendant cinq ans, au plus tard jusqu'à ce que son titulaire ait 70 ans [6-8].

\section{Prolongation de la reconnaissance}

Les médecins de niveau 1 doivent certifier tous les cinq ans à l'autorité cantonale qu'ils disposent des connaissances les plus récentes. Afin de tenir leur savoir à jour, les médecins des niveaux 2 et 3 doivent suivre, tous les cinq ans, une formation continue de 4 heures relevant de la médecine du trafic (refresher). Les spécialistes en médecine du trafic SSML doivent remplir les conditions de conservation de leur titre conformément au règlement régissant le titre [9].

\footnotetext{
Correspondance: SSML Centre Suisse de formation pour les expertises en aptitude à la conduite automobile de la Société Suisse de Médecine Légale Winterthurerstrasse 190 CH-8057 Zurich info[at]medtraffic.ch
}

\section{Dispositions transitoires}

Dans certains cantons, des formations organisées par la SSML ont eu lieu dès 2010. Les médecins qui ont déjà suivi des cours et obtenu dans ce cadre un niveau de qualification par les autorités cantonales conservent celui-ci comme auparavant. Celui-ci restera valide jusqu'au 30.6.2021 et ce n'est qu'à partir de cette date qu'une formation continue obligatoire deviendra nécessaire. Autrement dit, la période de validité ne commence à courir qu'avec l'entrée en vigueur de la nouvelle législation le 1.7.2016.

Le législateur a également prévu d'autres délais de transition [10]:

- Jusqu'au 31.12.2017, les médecins de niveau 1 seront encore autorisés à procéder tous les 2 ans aux examens des conducteurs âgés de plus de 70 ans selon l'ancien droit, sans la reconnaissance de l'autorité cantonale.

- Les expertises d'un médecin ou d'un psychologue du trafic conformes à l'ancien droit seront reconnues dans tous les cantons jusqu'au 31.12.2018, si elles ont été faites par un service désigné par l'autorité cantonale et ne sont pas antérieures à une année.

- Les modules 4 à 6 de la formation continue en médecine du trafic de la SSML suivis à partir du 1.7.2010 sont pris en considération dans le cadre de la reconnaissance.

- Les autorités cantonales pourront, jusqu'au 31.12.2019, confier la réalisation des examens relevant de la médecine du trafic qui doivent être réalisés par un médecin de niveau 4 à des médecins qui n'ont pas obtenu la reconnaissance prescrite ou reconnaître leurs résultats d'examen, si la personne a déjà procédé auparavant aux examens en question et si la personne à examiner devrait attendre trop longtemps avant de pouvoir subir l'examen en raison d'effectifs insuffisants chez les médecins ayant obtenu la reconnaissance correspondante (clause dite «de ventilation»).

$\mathrm{Au}$ terme des délais de transition correspondants, la nouvelle législation sera définitivement applicable. En cas de doute, le médecin concerné devra prendre contact avec l'autorité cantonale compétente.

\section{Remerciements}

Nous tenons à remercier l'asa pour sa collaboration agréable et constructive.

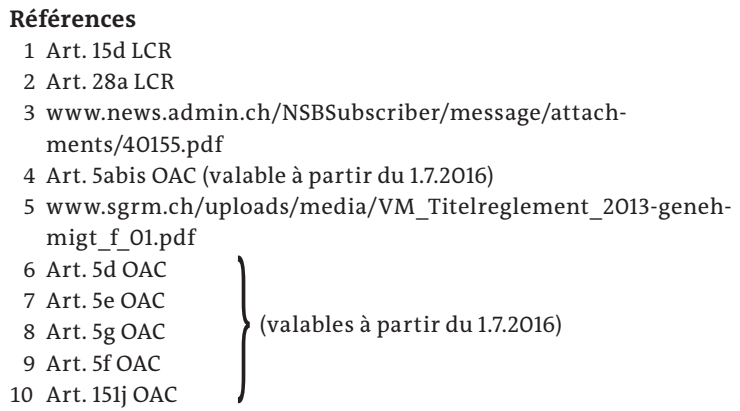

